\title{
1D geological imaging of the subsurface from geophysical data with Bayesian Evidential Learning.
}

$\underline{\text { Authors }}^{1}:$

Hadrien MICHEL ${ }^{1,2,3}$, Frédéric NGUYEN ${ }^{1}$, Thomas KREMER ${ }^{1}$, Ann ELEN ${ }^{4}$, Thomas HERMANS $^{2}$

(1) University of Liège, Urban and Environmental Engineering Department, Faculty of Applied Sciences, Liège, Belgium, (2) Ghent University, Department of Geology, Ghent, Belgium, (3) F.R.S.-FNRS (Fonds de la Recherche Scientifique), Brussels, Belgium, (4) KU Leuven, Department of Earth and Environmental Sciences, Leuven, Belgium

Corresponding author:

Hadrien MICHEL

University of Liège

Allée de la découverte, 9

B-4000, Liège (Belgium)

E-mail: hadrien.michel@uliege.be

Declaration of interest: none

Link to the code: https://github.com/hadrienmichel/BEL1D

Highlights:

- A new approach for 1D imaging from geophysical data.

- A set of MATLAB graphical user interfaces is provided.

- Estimation of the uncertainty on the model parameters.

- Consistent results for SNMR.

- BEL1D can be generalized to any type of $1 \mathrm{D}$ geophysical data.

${ }^{1}$ Authorship statement: Hadrien Michel developed the BEL1D codes and produced the results presented in this paper. Thomas Kremer has significantly contributed and improved all the SNMR related elements developed in this paper. The Mont Rigi SNMR dataset was acquired and pre-processed by Ann Elen for her Master Thesis. Frédéric Nguyen and Thomas Hermans both co-supervised the whole work and are at the basis of methodological development. 
Abstract:

Imaging the subsurface of the Earth is of prime concern in geosciences. In this scope, geophysics offers a wide range of methods that are able to produce models of the subsurface, classically through inversion processes. Deterministic inversions lack the ability to produce satisfactory quantifications of uncertainty, whereas stochastic inversions are often computationally demanding. In this paper, a new method to interpret geophysical data is proposed in order to produce 1D imaging of the subsurface along with the uncertainty on the associated parameters. This new approach called Bayesian Evidential Learning 1D imaging (BEL1D) relies on the constitution of statistics-based relationships between simulated data and associated model parameters. The method is applied to surface nuclear magnetic resonance for both a numerical example and field data. The obtained results are compared to the solutions provided by other approaches for the interpretation of these datasets, to demonstrate the robustness of BEL1D. Although this contribution demonstrates the framework for surface nuclear magnetic resonance geophysical data, it is not restricted to this type of data but can be applied to any $1 \mathrm{D}$ inverse problem.

Keywords: 1D imaging, uncertainty, Bayesian Evidential Learning, machine learning, SNMR, MATLAB. 


\section{Introduction}

Imaging the subsurface of the Earth through geophysical methods allows reducing costly invasive investigations or is sometimes the only way to investigate the Earth. Behind the geophysical images are models conceptualized in terms of physical properties (density, seismic velocities or resistivity) which may be converted to properties of interests (hydraulic conductivity, porosity or temperature) using petrophysical relationships. Decision-making, whether planning additional investigations for a scientific project, exploiting a geo-resource, or constructing a building, is often a driver for geophysical methods (Lawyer et al., 2001). Uncertainty assessment on those geophysical models is therefore a key concern to geophysicists, as it provides a much more consistent information in decision-making processes (Scheidt et al., 2018).

Computation methods to process geophysical data into models (other than simple mapping of the data) are either deterministic, often using the regularization approach, or stochastic, often using the Bayesian framework. Deterministic methods are limited in terms of uncertainty quantification and often rely on linear data error propagation based on a linearized approximation if needed (Aster et al., 2013; Kemna et al., 2007). On the other hand, stochastic methods may compute sets of models based on numerous inversions of perturbed datasets, or on the exploration of a prior model space using Markov chain Monte Carlos (McMC) methods (Aster et al., 2013; Sambridge, 2002).

Recently, due to the rise of computer performances, stochastic methods have been applied to geophysics and are able to quantify more properly uncertainty. Most algorithms rely on Markov chains Monte Carlos (McMC) methods in order to explore the prior model space (Sambridge, 2002). Those methods offer the advantage to provide a much more complete appraisal of the ensemble of possible solutions. Nevertheless, stochastic methods are often 
linked to high computation costs, since they require a large number of forward model runs to sample the posterior distribution in the prior model space, explaining why they are still often limited to scientific or numerical studies (e.g., Irving and Singha, 2010; Trainor-Guitton and Hoversten, 2011). Approximation of the posterior can be obtained (under some assumptions) through ensemble-based inversion (e.g., Bobe et al., 2019). Even more recently, machine learning also emerged as a potential contender to replace the inversion process, typically yielding to deterministic-like results due to the nature of neural networks and such (e.g., Laloy et al., 2019, 2017; Yang and Ma, 2019).

In this paper, we present Bayesian Evidential Learning (BEL) (Scheidt et al., 2018) as an innovative method for the unidimensional geophysical imaging of the subsurface. Bayesian Evidential Learning (BEL) is a general framework to handle data in geosciences, from the optimization of the data acquisition to the data interpretation, prior falsification, global sensitivity analysis, and the prediction of relevant model responses (Scheidt et al., 2018).

We will refer to the approach presented here as Bayesian Evidential Learning 1D imaging (BEL1D). It uses statistics-based relationships between forecast variables and data learned from realizations sampled from a prior distribution. As opposed to inversion methods, this approach does not require the time-consuming inversion of the dataset, but rather numerous (and parallelized) runs of the faster forward model. So far, BEL has been used to predict the dynamical response of subsurface models from well monitoring data (Satija and Caers, 2015), push-pull tests (Hermans et al., 2019) or time-lapse geophysical experiments (Hermans et al., 2018), i.e. model predictions mostly characterized by smooth variations in time and/or in space. BEL was also applied to show the non-uniqueness of the gravity inverse problem (Phelps et al., 2018).

In this contribution, we extend BEL to $1 \mathrm{D}$ geophysical imaging. Such $1 \mathrm{D}$ imaging arises in many geophysical methods where forward models assume a succession of homogeneous 
horizontal layers such as surface nuclear magnetic resonance (SNMR e.g., Behroozmand et al., 2015), surface seismic waves (e.g., Socco et al., 2010), electromagnetic surveys (e.g., Hanssens et al., 2019; Li et al., 2018), or vertical electrical sounding (e.g., Jha et al., 2008).

The main contributions of this paper compared to previous works are:

1) The application of BEL1D for static geophysical imaging. Here, we predict directly the posterior distribution of spatially distributed geophysical model parameters.

2) The estimation of the data-prediction relationship using kernel density estimation instead of Gaussian regression, because the former can be applied when the relationship deviates from linearity and Gaussian assumptions.

3) The validation of the approach by comparing it with state-of-the-art McMC

4) The link made between distance-based global sensitivity analysis and the canonical correlation analysis, yielding similar results.

5) The analysis of the number of samples in the prior.

This paper first introduces our implementation of BEL: Bayesian Evidential Learning 1D imaging (BEL1D). The method is then validated within a numerical example. To demonstrate the broad applicability of our approach, we also provide a validation within a nongeophysical related inverse problem (supplementary material: "Testing BEL1D on a synthetic mind experiment: oscillations of a pendulum"). Then, we present an example of BEL1D applied to field data, providing evidence that the method is already mature. Finally, we discuss on elements governing the accuracy of BEL1D, such as the number of models sampled in the prior and the choice of the bandwidth for kernel density estimation. 


\section{BEL for 1D imaging: methodology}

In this work, the focus is on computing geophysical models and assessing the uncertainty of the inferred models (Phelps et al., 2018). This interpretation part of BEL is also known as the prediction-focused approach (PFA), introduced by Scheidt et al. (2015). Contrary to deterministic approaches, this method does not rely on the stabilization of the ill-posed inverse problem, such as regularization for example, that imposes non-realistic constraint to the solution. It rather relies on the constitution of statistical relationships between predictors (the set of parameters of interest to the end-user) and data. These relationships are originating from models and (numerical) simulations of the geophysical problem that reflect the available prior knowledge.

BEL has already been applied previously to geophysical data - time-lapse electrical resistivity - by Hermans et al. (2016). Hermans et al. (2016) demonstrated the applicability of BEL to predict variations of subsurface physical properties with time-lapse electrical resistivity data. More broadly, they also demonstrated that BEL was a possible tool for geophysicists.

However, Hermans et al. (2016) presented a scheme that required numerical simulations of groundwater flow and transport and petrophysical relationships specific to hydrogeophysical monitoring (i.e. time-lapse data), limiting the extend of the geophysical prior model. Solving the time-lapse problem in geophysics is easier because we can invert for changes in the model (e.g., Kemna et al., 2002; Nguyen et al., 2016). In Hermans et al., (2016), a petrophysical relationship and the background distribution of resistivity were taken as known, which simplified the inference of the posterior distribution. Static data are also characterized by a higher noise level and thus more challenging to process (LaBrecque et al., 1996; Lesparre et al., 2017). This is also the first application where BEL is used to directly estimate spatially distributed model parameters. In our case, the forecast variables are static geophysical models, 
95 directly linked to the geophysical experiment, hence no petrophysical relationship is required.

96 Such situations generally bear more prior uncertainty and wider variability. Moreover, our aim

97 is to propose a fast generation of subsurface models along with uncertainties. The adapted 98 BEL1D method consists of six steps (Figure 1):

1. Definition of the prior uncertainty based on prior field knowledge, generation of prior models (yellow box in Figure 1) and associated data (blue box in Figure 1) by forward modeling

2. Reduction of the dimensionality of the data using principal component analysis (PCA)

3. Constitution of statistical relationships between the model parameters and the reduced data (green cloud in Figure 1), using canonical correlation analysis (CCA)

4. Generation of posterior distributions for the model parameters in reduced space by constraining the bivariate distributions to field data (red box in Figure 1) using kernel density estimators (contrary to previously used Gaussian process regression (Hermans et al., 2016))

5. Sampling of the constituted (non-Gaussian) distributions

6. Back-transformation of the samples into the original space, delivering a set of 1D models of the subsurface that are constrained to the knowledge of the geophysical data (purple box in Figure 1).

Each step is explained in detail in the following sections. 
Prior

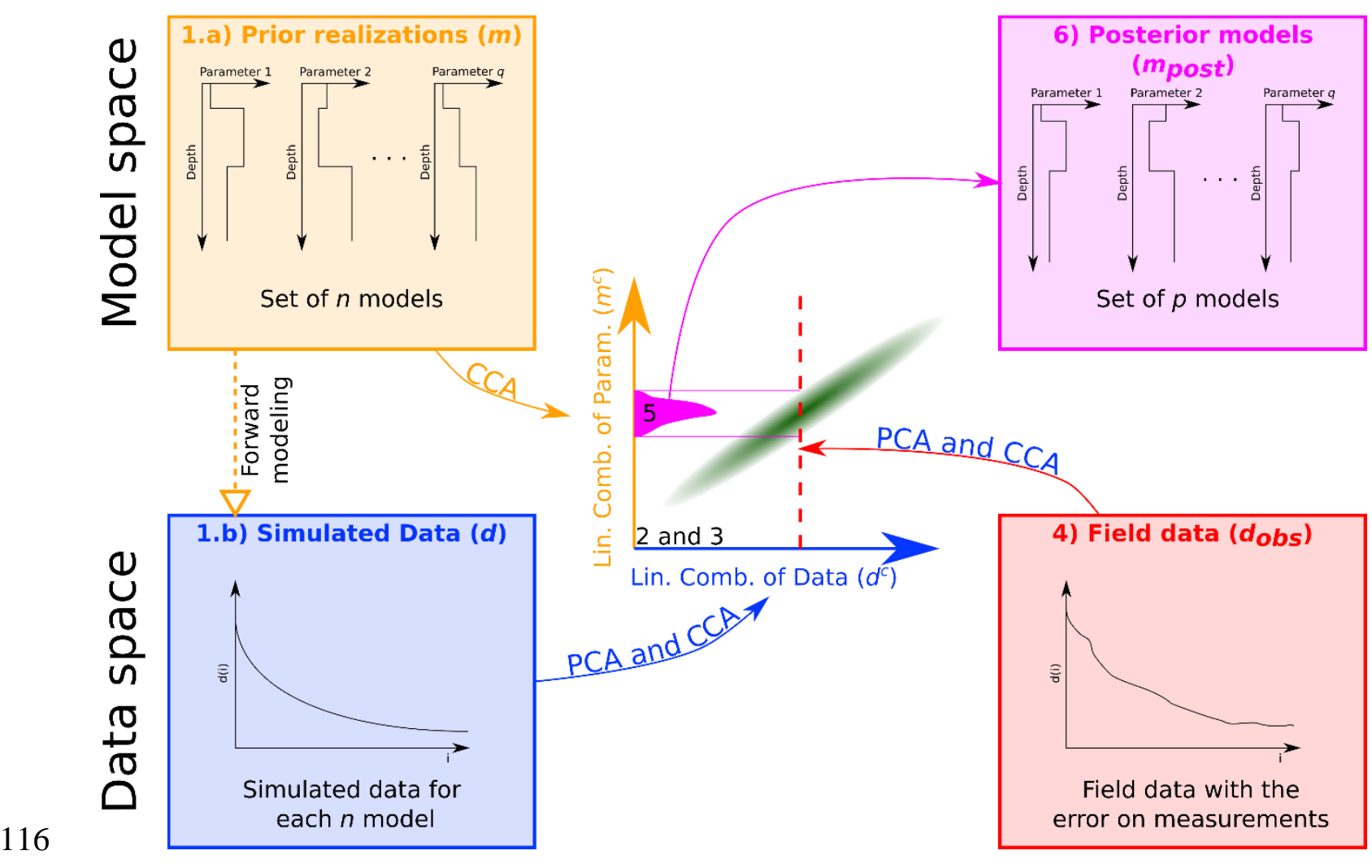

Although BEL1D can be applied to any 1D inverse problem, for the clarity of the paper, we briefly introduce SNMR, the specific method chosen to illustrate the BEL1D method. SNMR is a geophysical technique dedicated to the detection and characterization of aquifers,

121 whose main advantage and particularity is that the measured signal (an exponential decay) is

122 directly linked to the amount of water present in the subsurface. For more details about the 123 nature and origin of the SNMR signal, we refer to Behroozmand et al. (2015). The main goal 124 of SNMR experiments is to retrieve the distribution of the water content and the relaxation time 125 with depth. The latter depends on the pore space geometry and can be related to hydrodynamic 126 parameters such as the porosity or the permeability. The acquisition of SNMR data on the field 127 consists in the injection of an alternative current in a transmission loop (typically circular or in 128 an eight-shape) directly followed by the measurement of the induced current in the reception 129 loop (often the same as the transmission loop). The injection is tuned at the resonance frequency 
of water (called the Larmor frequency), perturbing the quantum state of protons contained in

131 water molecules. Once the injection is stopped, the protons are relaxed back to their original

132 state, inducing a current with a decreasing amplitude with time in the receiver loop. This

133 response is called the free-induction decay (FID) and constitutes the collected data. The

134 injection is repeated with different pulse moments, sounding different zones of the subsurface.

135

136

137

138

139

140

\subsection{Step 1: prior realizations and associated data}

In BEL1D, the models are described in a classic manner with a finite number of layers $N_{L}$, the last one being a half-space. For each layer, the $N_{P}$ physical properties related to the geophysical experiment and layer thickness are assigned. This means that a model can be described with a low number of parameters: $\left(N_{L} \times\left(N_{P}+1\right)\right)-1=q$. Defining the prior model space $f_{H}(\boldsymbol{m})$, i.e. the space describing the prior knowledge of the subsurface physical properties before data acquisition, consists in assigning a distribution to the values of each of those $q$ parameters. The shapes of those distributions are unconstrained and must best represent the prior knowledge of the survey site, either originating from previous experiments or from general geological and geophysical considerations. Once the prior model space is defined, we generate random models within the prior boundaries, hereafter called prior realization: $\boldsymbol{m}(q \times 1) \in f_{H}(\boldsymbol{m})$.

For example, the prior model space for SNMR experiments is defined using both the water content (in \%) and relaxation time (in ms) distributions along depth. This leads to a finite number of layers, of unknown thickness, and of unknown water content and relaxation time, thus to the description of a model with only five parameters for a 2-layer model.

For all prior realizations, we compute their associated response using the same acquisition parameters as the experimental conditions. Doing so, we produce the synthetic data 
153 vector: $\boldsymbol{d}(l \times 1)$. To perform this operation, we use the (non-linear) forward operators $(K)$ of the geophysical problem of interest such that

$$
\boldsymbol{d}=K(\boldsymbol{m})
$$

For more details about the forward operator associated with the SNMR method, we refer to Hertrich et al. (2007). Often, the dimensionality of the data $(l)$ is relatively large. For SNMR, the data space is composed of tens of time-decaying signals (one for each pulse moment) with sampling rates on the order of $100 \mathrm{~Hz}$ measured for about 1 seconds, thus resulting in several thousands of dimensions. and the "data space" which contains the synthetic geophysical data sets associated with each model. To explore the statistical relationships that exist between these two spaces, one must first ensure that their dimensionality is sufficiently low (typically about 10 dimensions per space) to allow for reasonable computational costs. In the case of SNMR, as in most other cases variability.

The PCA method seeks for linear combinations of variables that maximize the

174 variability in the first dimensions using eigenvalue decomposition (Krzanowski, 2000). This 175 operation results in PCA scores, the values of the transformed data. This way, the first 
amount of the variability. Those later can be discarded, to gain memory and ease following computations. In the SNMR context, the same process is applied to the original data space and performs very well since the dimensions are often highly correlated. The obtained reduced data space has a dimensionality $k \ll l$ and is noted $\boldsymbol{d}^{f}(k \times 1)$. Note that in other geophysical problems, this PCA step may not be necessary if the dimensions of both the model and data space are low or may also be necessary for both the model and data spaces if their dimensions are too high. Noise analysis using Monte Carlo simulations (Hermans et al. , 2016) provides covariance between the different PCA components representing the data $\left(C^{f}\right)$. This translates in uncertainties on the PCA scores of the test data. The detailed procedure for noise propagation is presented in subsection 2.7 .

2.3. Step 3: constitution of statistical relationships between the models parameters and the reduced data, using canonical correlation analysis BEL1D relies on the constitution of statistics-based relationships between earth models and simulated data. This step is the core of BEL1D. Several methods are suitable to derive such a relationship, but we choose canonical correlation analysis (CCA) for its simplicity. In essence, CCA transforms the (possibly PCA-reduced) model space $\left(\boldsymbol{m}^{f}\right)$ and the PCA data space $\left(\boldsymbol{d}^{\boldsymbol{f}}\right)$, so that the canonical correlation (an approach formula of the correlation) between the resulting CCA spaces ( $\boldsymbol{m}^{\boldsymbol{c}}-$ Equation $2-$ and $\boldsymbol{d}^{\boldsymbol{c}}-$ Equation 3) is maximized (Krzanowski, 2000). Each of the dimensions of the CCA spaces are orthogonal, meaning that there is no redundancy between the dimensions. We obtain:

$$
\begin{aligned}
d^{c} & =d^{f} A^{T} \\
m^{c} & =m^{f} B^{T}
\end{aligned}
$$

where $\boldsymbol{A}$ is a matrix of dimensions $m \times k$ and $\boldsymbol{B}$ is a matrix of dimensions $m \times q$ ( $m$ is the minimum between $k$ and $q$ ). Graphically, we can observe the statistical relationship between $\boldsymbol{d}^{\boldsymbol{c}}$ and $\boldsymbol{m}^{\boldsymbol{c}}$ as both spaces have the same dimensions $(1 \times m)$. As long as $k$ is larger than $q$, it 
is possible to back-transform the models from the CCA model space to the original model space using the inverse of $\boldsymbol{B}$ (Hermans et al., 2016). space using kernel density estimators

In canonically correlated space, the correlation between dimensions is maximized.

Hence, it is possible to produce a meaningful statistical description of the bi-variate distribution.

This enables to determine a posterior distribution in the CCA model space constrained by the knowledge of field data. This posterior distribution represents the probability density function

corresponding to the observed data $f_{H}\left(\boldsymbol{m}^{c} \mid \boldsymbol{d}_{\text {obs }}^{c}\right)$, we use a kernel density estimator (KDE) with

210 a Gaussian kernel (Wand and Jones, 1993) similar to Hermans et al. (2019) where they faced non-linearity issues when validating BEL for hydrogeology. Gaussian regression (Hermans et al., 2016; Satija and Caers, 2015) was tested; but in many cases, CCA did not yield perfectly linearly correlated relationships with Gaussian prior distributions, two necessary conditions to apply Gaussian regression. We thus implement KDE for its robustness to estimate posterior distribution in a large variety of situations. KDE computes, for a given location in space, the sum of the contribution of each point from the CCA space. We use a multi-Gaussian kernel centered on the points with bandwidths chosen accordingly to the point density (see subsection 5.2). The resulting distributions in the CCA model space are not constrained to any given distribution of known shape. The process is illustrated in the center of Figure 1.

\subsection{Step 5: sampling of the constituted distributions}

The posterior distribution is not constrained to a known distribution. Therefore, sampling is done through the inverse transform sampling method (Devroye, 1986). This sampling procedure benefits from the properties of the cumulative distribution function that 
224 links a uniformly distributed variable to any distribution. The sampler thus transforms a value 225 sampled in a uniform distribution to the corresponding value in the original distribution via the 226 inverse of the cumulative distribution function. We can now easily generate a set of samples 227 from the posterior distributions in the reduced space:

$$
\boldsymbol{m}_{\text {post }}^{c} \in f_{H}\left(\boldsymbol{m}^{c} \mid \boldsymbol{d}_{\text {obs }}^{c}\right)
$$

\subsection{Step 6: back-transformation of the samples into original space}

The set of samples in CCA model space can be back-transformed into the original model space, using the inverse of the $\boldsymbol{B}$ matrix $(q \times q)$. We thus have the posterior in the original 231 physical space:

$$
m_{\text {post }}=m_{\text {post }}^{f}=B^{-1} m_{p o s t}^{c}
$$

Here, in the SNMR context, since the model space was not reduced using principle component analysis, the models in PCA space are the same as the ones in original space. Because Gaussian regression or KDE with Gaussian kernel has no limit on the value of sampled parameters, a few samples might occasionally be located outside the prior model space, in particular when the model is at the extremity of the prior. Those models are removed from the sampled space as they do not correspond to the definition of the problem.

\subsection{Noise propagation in BEL1D}

In order to investigate the effect of noise on BEL1D, one can analyze the variation of the PCA scores to the estimated field noise level (if the latter can be estimated). If the scores of the first $k$ components are not varying in a significant way, then, one can assume that noise has 242 no impact on the imaging process. On the other hand, if the noise impacts the scores 243 significantly, BEL1D considers this impact by propagating uncertainty (represented by $C^{f}$ in 244 Equation 6) on the reduced observed data in the canonically correlated space, leading potentially to more complex computation of the posterior distributions (Hermans et al., 2016). 
246 This uncertainty is propagated in the CCA dimension according to linear error propagation from 247 equation (2):

$$
C^{c}=A C^{f} A^{T}
$$

To estimate $C^{f}$, we explore the PCA scores and their modifications when noise is 249 present in the dataset. To do so, we take the data associated to each of the prior realizations as 250 a basis and compute the PCA scores when one model is perturbed with a noise level similar to 251 the data. The operation is repeated for a significant sub-sample (in the latter example, we used 25250 randomly selected models) of the prior samples allowing to derive the PCA score covariance 253 matrix $C^{f}$.

Contrary to Gaussian regression where the addition of covariance to the observed reduced data only slightly increases the complexity of the formula for the sampling parameters 256 (Hermans et al., 2016), the addition of uncertainty on the reduced observed data highly hinders performances of the Kernel density estimation. Assigning uncertainty to the observed data $(\mathrm{X}$ in Figure 2) requires the computation of the kernel density estimation of the reduced posterior 259 distributions for multiple sets of randomly sampled $d_{o b s}^{c}+\epsilon$, then processing to sampling and 260 back transformation from those sets of distributions to constitute models of the posterior 261 distributions. This can be avoided by enlarging the bandwidth of the kernel density estimator 262 (Bowman and Azzalini, 1997) in the X direction (in the CCA data space) according to $C^{c}$. This 263 accounts for the uncertainty on the exact position of the data in the reduced space and therefore 264 account for noise, without adding complexity to the computations, as is shown in Figure 2. 

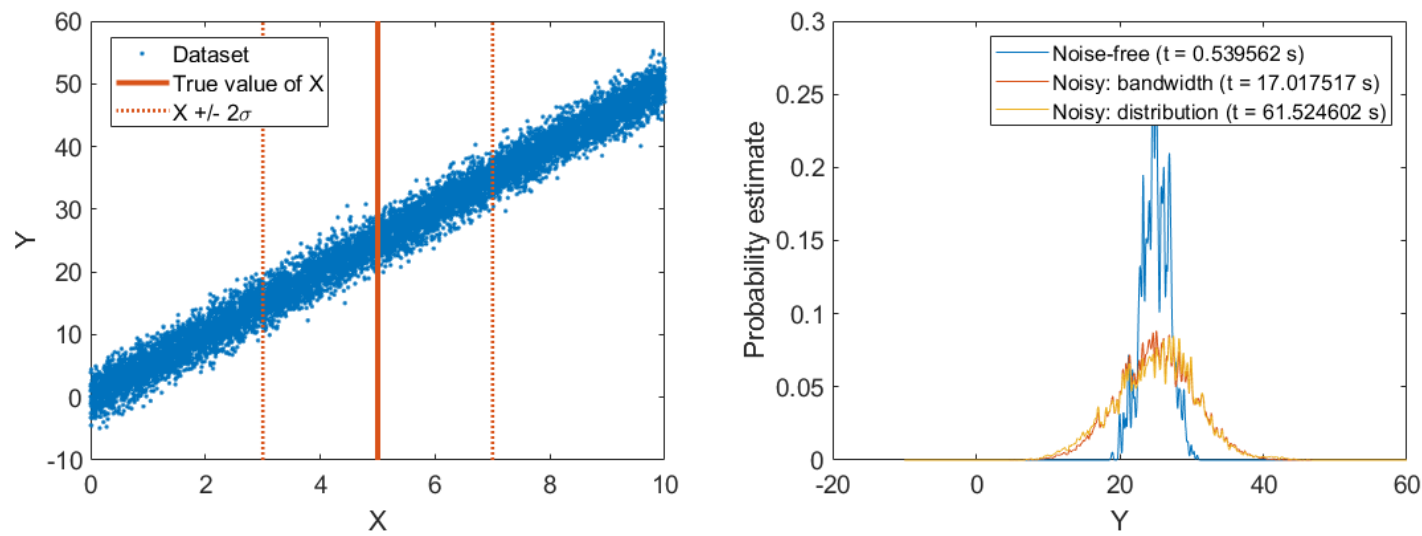

Figure 2 : Example of the effect of noise on the computation of the kernel density estimator for the probability density functions. On the left, a random dataset that is highly correlated and the associated true value of X. On the right, the estimated distributions for the values of $Y$ constrained to the known value of $X$ and its associated error of 1. The computation time tis given for the different cases.

\section{Numerical example}

In this section we will present results obtained by applying BEL1D to numerical layers: a first unsaturated layer and a saturated half-space (Table 1). A more complex case is presented in section 4, and a non-geophysical related inverse problem is presented in supplementary material ("Testing BEL1D on a synthetic mind experiment: oscillations of a pendulum").

\begin{tabular}{|c|c|c|c|}
\hline & Thickness (m) & Water content (\%) & Relaxation time (ms) \\
\hline Layer 1 & 5 & 5 & 100 \\
\hline Half-space & $/$ & 25 & 200 \\
\hline
\end{tabular}

Table 1: Characteristics of the synthetic model

\subsection{Computation of the model space and data space}

The data are simulated for SNMR using the MRSMatlab toolbox (Müller-Petke et al., 
loops have a $50 \mathrm{~m}$ diameter with 2 turns). To simplify the computations, the earth is set as

282 resistive, neglecting the effect of electrical conductivity in signal attenuation and phase shift.

283 Therefore, the signal is purely real. However, the process presented in this paper is not limited

284 to real-valued data. SNMR is known to be impacted significantly by electromagnetic (EM)

285 noise (e.g., Behroozmand et al., 2016). Different types of EM noise can affect the SNMR data.

286 However, when most of these components have been filtered out, the SNMR signal will remain

287 contaminated by a Gaussian noise distribution, whose magnitude cannot be reduced further. To

288 represent this, we chose to add a Gaussian noise level to the dataset, corresponding to a mean

289 signal-to-noise ratio of $10(35 \mathrm{nV})$, which is a relatively high value in usual SNMR surveys.

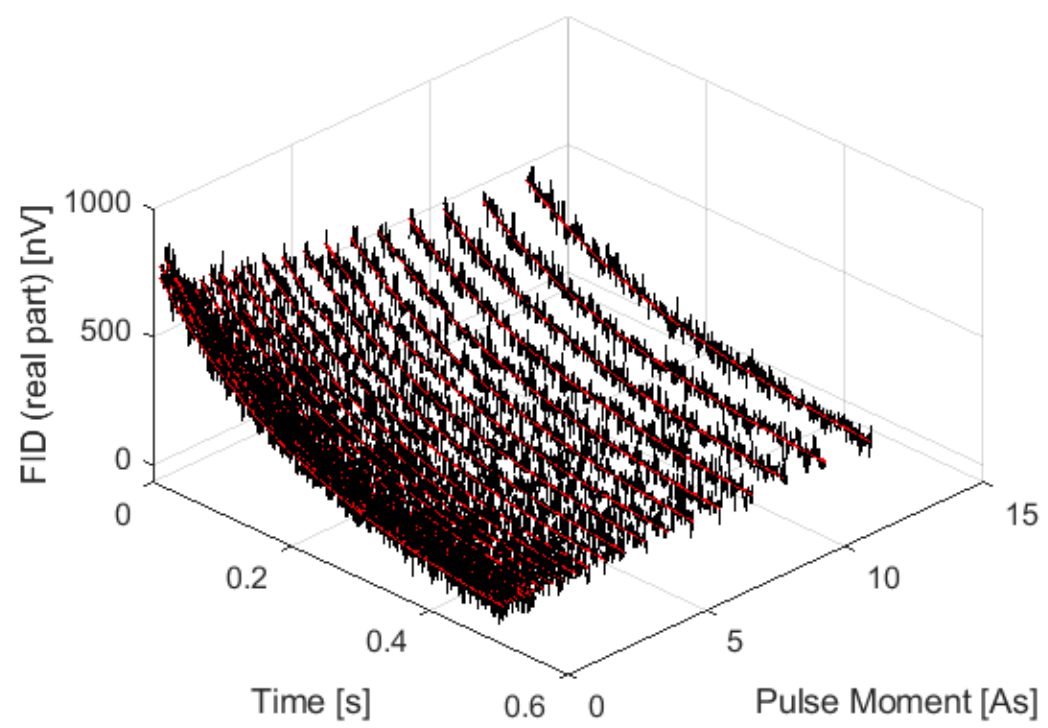

Figure 3 : Simulated data for the SNMR experiment. The black curves represent the noisy dataset and the red curves the noise-free one.

As can be observed in Figure 3, the initial amplitude of the signal is around $500 \mathrm{nV}$, and signal-to-noise ratio of 10 produces quite noisy signals, but the decaying behavior remains observable.

The prior model space reflects the prior knowledge of the study site. In our case, we will assume that it is known that the subsurface is composed of one layer resting over an infinite 
298 half-space. Then, the rest of the properties will be defined as uniformly distributed variables 299 varying in the intervals described in Table 2 . The prior intervals are not necessarily centered on 300 the actual value of the parameter. This is to better simulate real situations, where the user will 301 only have rough information about the subsurface constitution. 5000 prior realizations are 302 generated using a Latin-hypercube sampler (McKay et al., 1979). The forward model that was 303 used to simulate the test data is reused here to generate the synthetic data set.

\begin{tabular}{|l|l|l|l|l|l|l|}
\hline \multirow{2}{*}{} & \multicolumn{2}{|l|}{ Thickness [m] } & \multicolumn{2}{l|}{ Water content [\%] } & \multicolumn{2}{l|}{ Relaxation time [ms] } \\
\cline { 2 - 7 } & Minimum & Maximum & Minimum & Maximum & Minimum & Maximum \\
\hline Layer 1 & 2.5 & 7.5 & 3.5 & 10 & 5 & 350 \\
\hline Half-space & $/$ & $/$ & 10 & 30 & 5 & 350 \\
\hline
\end{tabular}

3.2. Global sensitivity analysis and dimension reduction

We performed a distance-based global sensitivity analysis (Park et al., 2016) whose 308 results are presented in Figure 4 (Left). The parameters corresponding to the first layer $\left(W_{1}\right.$ 
310 should therefore lead to a poorly reduced uncertainty on those parameters.
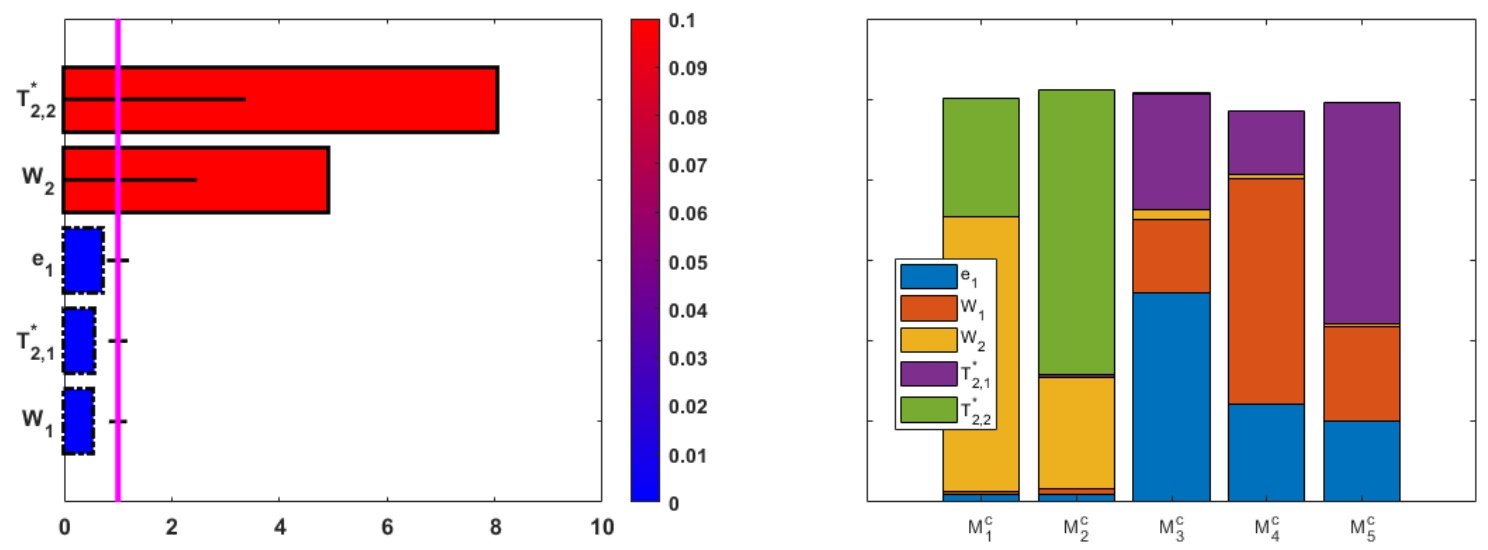

312 Figure 4 : Left: multivariate sensitivity analysis performed on the simulated data (the red bars correspond to parameters that have an effect on the data and the blue ones to parameters that have a negligible effect). Right: combinations of parameters representing the canonically correlated model space dimensions (the values are scaled for visual purposes)
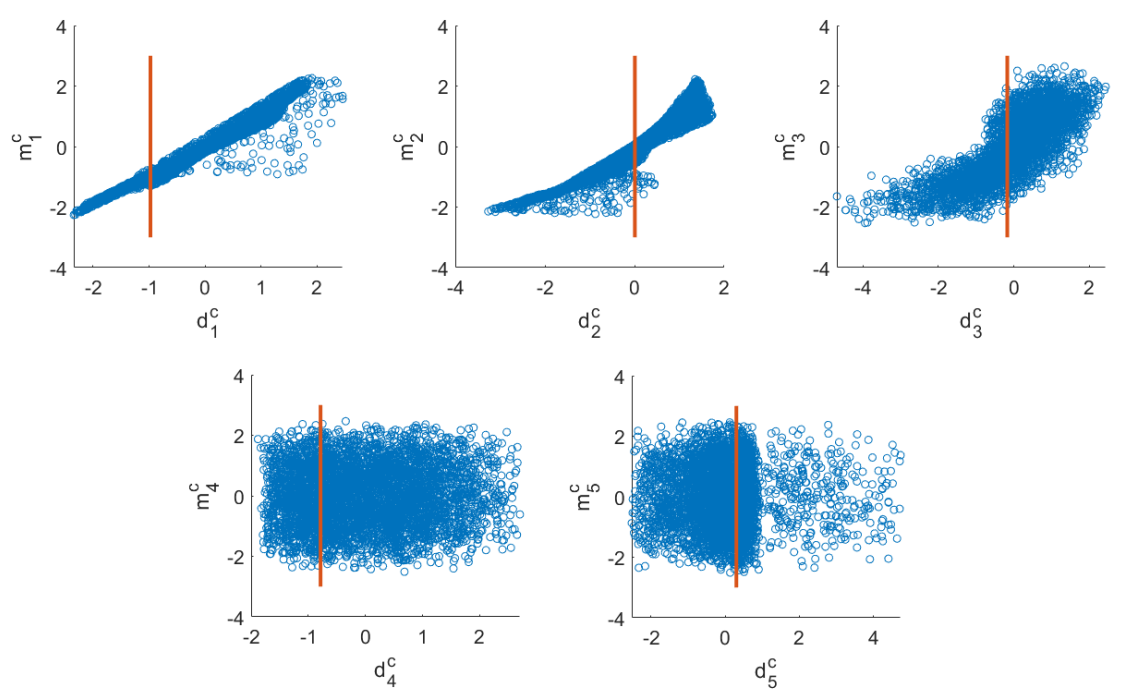

Figure 5 : SNMR - Data space versus model space in the canonically correlated space. The blue dots represent 318 the prior models and data spaces and the orange line represents the exact value of the test data in the reduced 
From the initial 11.904 dimensions in the dataspace, PCA enabled a reduction to only 5

321 dimensions, while managing to keep more than $90 \%$ of the variability. The canonical correlation analysis is shown in Figure 5. It is observed that (1) the correlation between the models and the data is larger for the first 3 CCA dimensions, (2) the correlation is not linear

324 from the $2^{\text {nd }}$ dimension and (3) from the $4^{\text {th }}$ dimension, the points are scattered and not 325 correlated. The fact that the last dimensions are scattered (note that each dimension is a linear 326 combination of the model parameters) informs us on the inability of the data to efficiently 327 predict all the parameters simultaneously. This is confirmed by the linear combinations that 328 constitute the CCA model space dimensions (Figure 4, Right): we observe that the three last 329 reduced dimensions are mainly linear combinations of the insensitive values (Figure 4, Left).

330 Note that non-linear statistical techniques (e.g., Gorban et al., 2008; Lawrence, 2012) could 331 potentially lead to better characterization of the data-model relationship. 

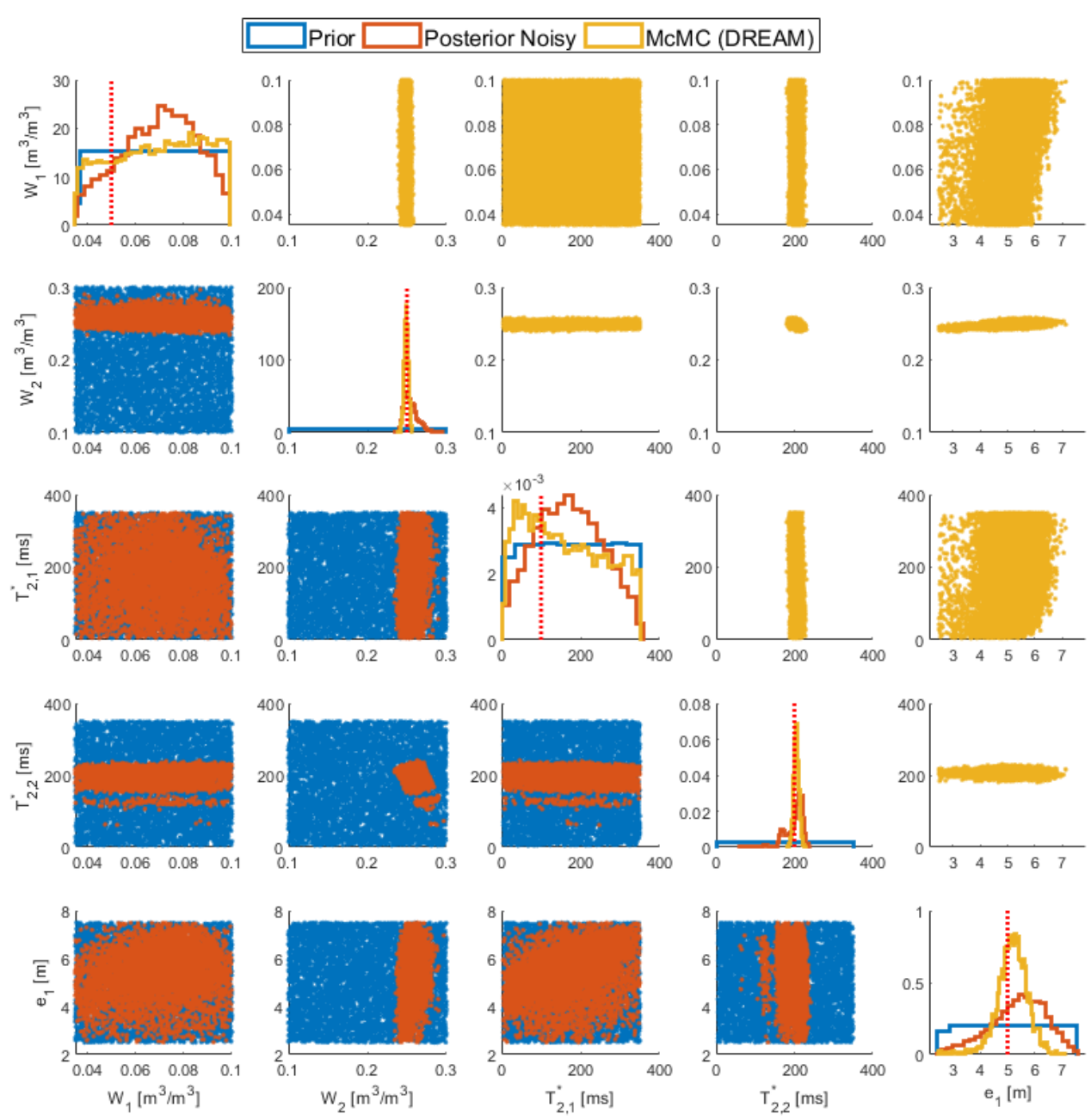

Figure 6: Results of the BELID process on the synthetic noise-free data from the 2 layers model. The obtained distributions are the posterior distributions estimated from the noisy dataset in red. The sampled prior model space is represented by the blue distributions. The test values are represented with dashed red lines in the histogram plots. Results of the McMC algorithm DREAM are presented in yellow.

BEL1D is applied to the simulated noisy dataset. Figure 6 shows the prior and posterior

339 distributions of parameters obtained through BEL1D. We observe that the uncertainty on the 340 half-space parameters is dramatically reduced through BEL1D. On the other hand, the 341 insensitive parameters $T_{2,1}^{*}$ and $W_{1}$ are poorly reduced. This insensitivity to some of the 342 parameters is also clearly identified in the pendulum example (Supplementary Material). As 
can be observed, the test value of the parameter is always contained in the posterior distribution.

344 The multivariate analysis of the parameters shows that most of the parameters are independent.

345 However, some correlations are observed for parameters that are related to the same layer $\left(T_{2,2}^{*}\right.$

346 and $W_{2}$ for example). Figure 7 (Left) presents another representation of the posterior results,

347 where the data RMS of posterior samples are color-coded, and where each model is a set of

348 linked parameters. The presented results show that a large part of the posterior models has a

349 very low RMS error. The trend in the water content set through the prior model space is

350 respected. In contrast, the trend for the relaxation time is not resolved by BEL1D. The analysis

351 of the RMS and the comparison with DREAM (Figure 7, Right) shows that is related to the

352 insensitivity of the data and not to the method itself.
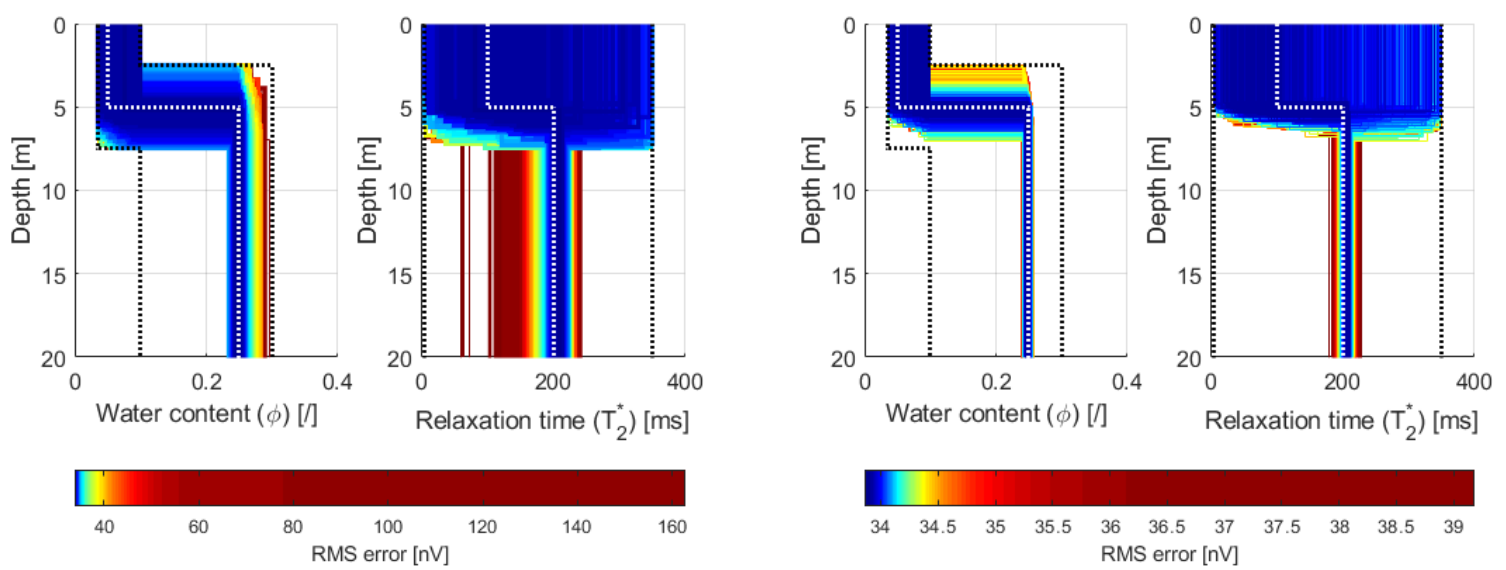

Figure 7 : Left: results of the BEL1D process on the synthetic noisy data from the 2 layers model. Each displayed model is presented with its associated RMS error value. In order to enhance visualisation, each colour is associated to the same number of models. The dashed white lines represent the test values and the dashed black lines represent the extent of the prior model space. The similar graph is also presented for DREAM results (Right).

We applied a Gaussian likelihood with 15 generations and 10000 chains and a burn-in period of 5000 chains. However, to achieve convergence towards reasonable uncertainties, changes to

362 the definition of the dataset are required. We had to use gate integration, similarly to what is 
applied in MRSMatlab (Müller-Petke et al., 2016) in order to attribute higher loads to the first

364 part of the decay curves, thus lowering the impact of noise on the estimation. For the presented results, we use 5 gates for each pulse moment. However, we must note that the results of DREAM are highly sensitive to the number of gates: with 1 or 2 gates, nearly no reduction of uncertainty is observed, whereas with a too large number of gates (to the limit with no gate integration) false posterior distributions are observed (the test values are outside of the predicted posterior). The results are presented alongside the BEL1D results in Figure 6. They show that BEL1D tends to have a slightly lower performance than DREAM. This is related to the approximation of the problem in a lower dimension space where the actual data misfit is never

\section{3 distribution (Figure 7).}
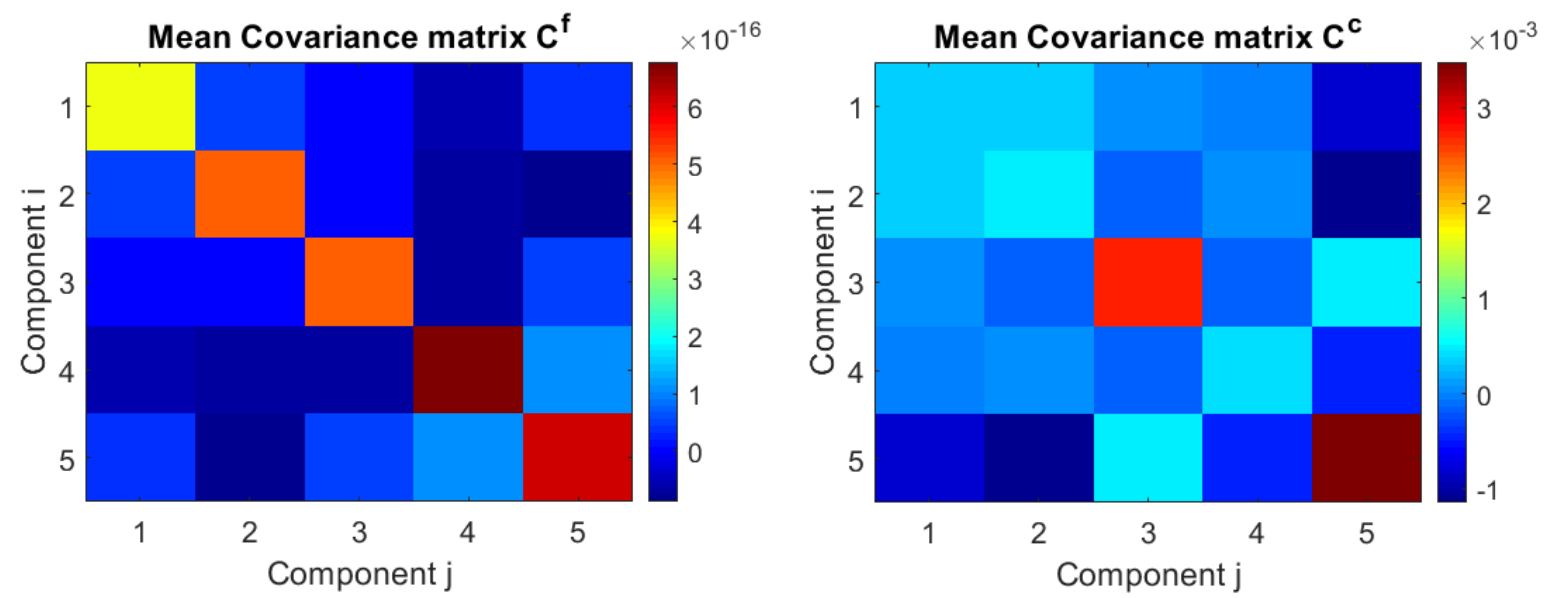

Figure 8: Impact of noise on the PCA scores (the first 5, kept after dimensionality reduction), covariance matrix for the PCA scores impacted by noise (left) and the corresponding covariance in CCA space (right). This first matrix is estimated through the mean of the covariance matrices observed between the noise-free scores and the noisy ones.

To estimate the posterior distributions, we needed to account for the impact of noise in the process. The matrix $C^{f}$ presented in Figure 8 shows very low values for the covariance.

381 However, once transformed into CCA space $\left(C^{c}\right.$, Equation 6), we observe that the effect of 
noise, even if limited, is still observable. From a default bandwidth of 0.01 in the $\mathrm{X}$ direction for the kernel density estimation, we computed bandwidths ranging from 0.017 to 0.059 .

Those increased bandwidths explain the presence of some outliers in the dataspace (Figure 7, LeftErreur ! Source du renvoi introuvable.). When observing the CCA space (Figure 5), it is noticeable that the second dimension (mainly representing the decay time of the second layer) shows models that are outside the main distribution but close to the position of the reduced test data. When using the bandwidth that takes into account the impact of noise, those models are englobed into the posterior. Nonetheless, those models correspond to large RMS error and are easily identified (Figure 7).

An example with more than two layers is presented in supplementary material (“Application of BEL1D to a four-layer model").

\section{Case study: Mont Rigi (Belgium)}

The Mont Rigi is located in the Belgian Fagnes region, in the Eastern part of the country. This site presents an ideal case study for SNMR as it is remote and far from any electromagnetic noise sources, due to its natural reserve classification.

Geologically, the site is characterized by a metric peat layer on top of a Cambrian bedrock (La Venne formation) known as an aquiclude with a very low water content (Gilson et al., 2017). In contrast, peat is known to present very high water contents with observed total porosities around 90\% (Wastiaux, 2008). According to previous GPR exploration (Wastiaux and Schumacker, 2003), the peat layer at the experimental site should have a thickness between 2.5 and 4.5 meters. The SNMR response should therefore allow to easily distinguish the two layers with a properly designed experiment. The designed on-site experiment consisted of a classical coincident loops transmitter/receiver couple with a diameter of 20 meters. 
The raw data have been preprocessed to lower the impact of noise with despiking, 406 harmonic modelling, reference noise cancellation and despiking again (Müller-Petke et al., 2016). The signals have been further truncated to 0.2 seconds to decrease the impact of noise, mostly present at large timeframes. The resulting noise amplitude is quite low for SNMR (18 $\mathrm{nV}$ ). Then, the signals have been inverted using the QT Inversion (Müller-Petke and Yaramanci, 2010), to constitute a benchmark to compare to the results of BEL1D. We used a smooth-mono 411 model description (smooth models with a single relaxation time for a given depth) and a regularization parameter of 6000 for both water content and relaxation time using the L-curve criteria (Aster et al., 2013). Then, BEL1D was applied with the uniformly distributed prior 414 model space described in Table 3. We sampled 5000 prior realizations and produced 5000 415 models for the posterior.

\begin{tabular}{|l|l|l|l|l|l|l|}
\hline & \multicolumn{2}{|l|}{ Thickness [m] } & \multicolumn{2}{l|}{ Water content [\%] } & \multicolumn{2}{l|}{ Relaxation time [ms] } \\
\cline { 2 - 7 } & Minimum & Maximum & Minimum & Maximum & Minimum & Maximum \\
\hline Layer 1 & 0 & 7.5 & 30 & 80 & 0 & 200 \\
\hline Layer 2 & $/$ & $I$ & 0 & 15 & 100 & 400 \\
\hline
\end{tabular}

418 of uncertainty for some parameters, as the first three dimensions show high correlations. The

419 first dimension, dominated by the water content of the half-space shows an especially narrow 420 relationship between the reduced data and model space. On the other hand, the last dimension, 421 mostly represented by the water content of the first layer, shows no specific correlation. We 422 should therefore expect a much better reduction for the parameters of the half-space than for 423 the first layer. 

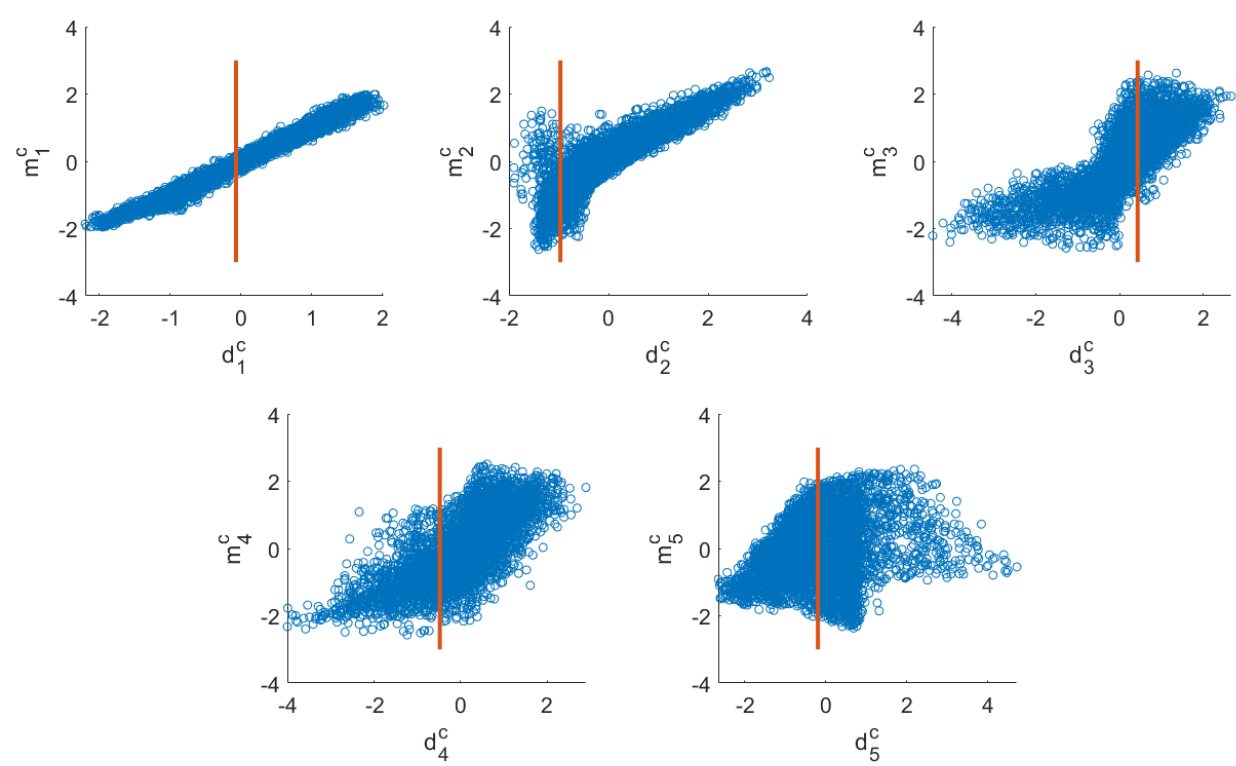

424

425

426

427

428

429

Figure 9: Mont Rigi-Data versus model space in the CCA space. The blue dots represent each prior realizations and the orange line the position of the field data.

The noise level (after denoising) impact on BEL1D was assessed and resulted in significant changes in bandwidths for the kernel density estimation, between 0.04 and 0.12 instead of the default 0.01 value. 

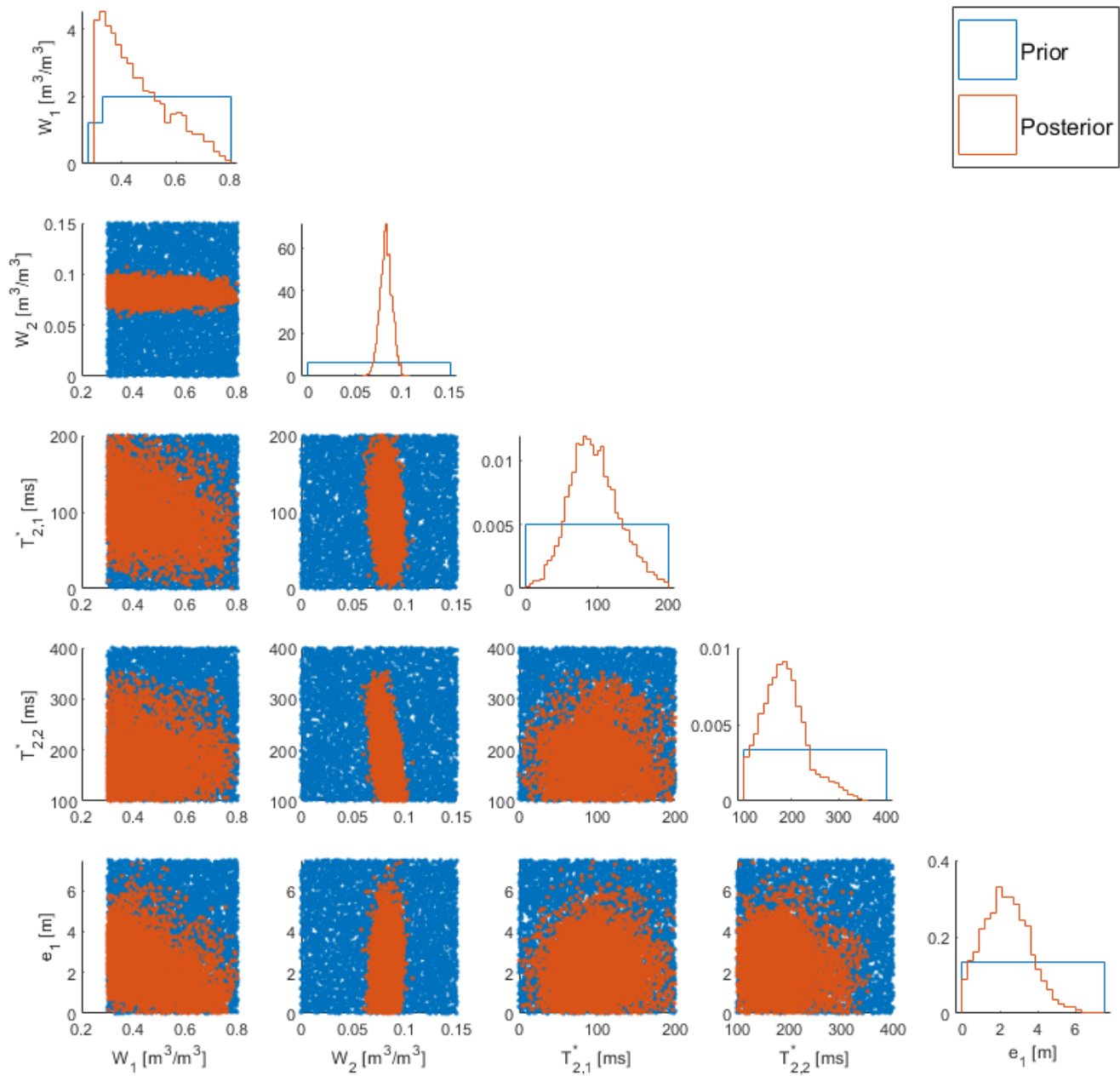

Figure 10: SNMR at Mont Rigi - Prior (blue) and posterior (orange) distributions of the parameters.

The analysis of the posterior parameters model space (Figure 10) shows that the

433 reduction of uncertainty from the prior model space to the posterior is significant and produces

434 consistent results, when compared to the geological context and the QT inversion solution

435 (dashed gray lines in Figure 11). The thickness of the peat layer tends to be smaller than the maximum estimate (4.5 m) from to the GPR interpretation (Wastiaux and Schumacker, 2003).

437 The use of a narrower prior taking into account the GPR information would prevent such a 438 behavior. This is illustrated by the decrease of the maximum value of the thickness for the first 
layer when the water content of the aforementioned layer increases. This behavior arises from

440 the non-uniqueness of the SNMR response stated above.

442 mean square error lower than the noise level in the dataset, implying that the set of probable

443 models provides an efficient estimation of the site parameters. The RMS errors of the posterior

444 samples show that they all explain the data to a similar level; the uncertainty is thus intrinsic to

445 the data set and the non-unicity of the solution. The non-uniqueness of the solution is easily

446 observable, with two distinct behaviors: one with a median water content and a thick first layer

447 and another with a very high water content but a thinner first layer. Those two cases show

448 similar RMS errors, hence are explaining the data at the same level. The probability of each

449 posterior model is shown in the RMS error colorbar for which each color represents the same

450 number of models. Therefore, it is observed that the low RMS error models also correspond

451 with the most probable ones.
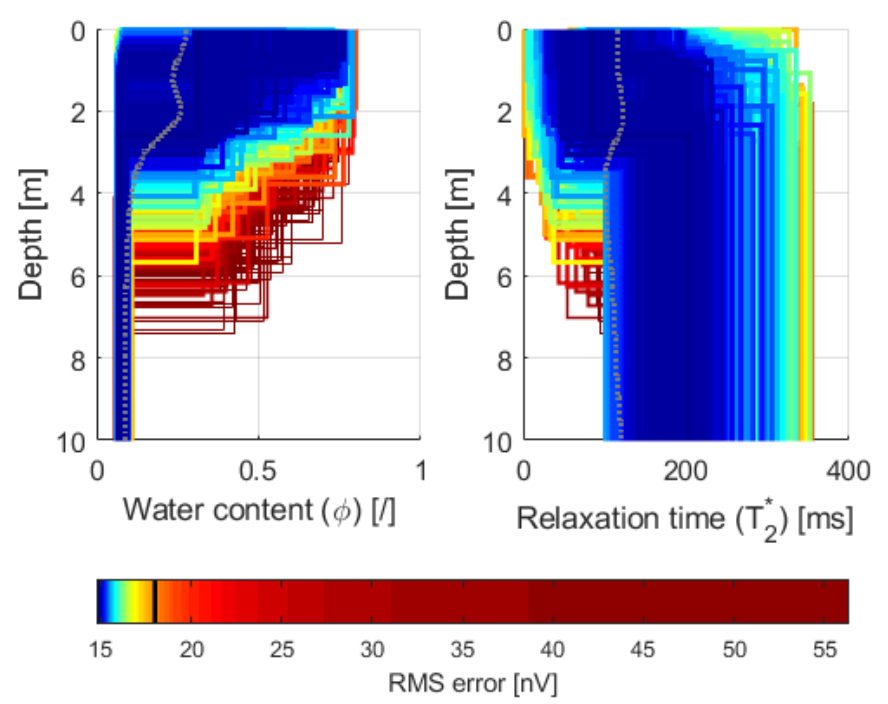

Figure 11: SNMR at Mont Rigi - Posterior models distributions with their associated misfit and results of the QT inversion (dashed gray line). The black line in the RMS error graph represents the level of noise in the original 


\section{Discussion}

457

458

459

460

461

462

463

464

465

466

467

468

469

\subsection{Impact of the number of sampled models in the prior model space}

The optimum number of models in the prior model space sample is a tradeoff between precision of the posterior models distributions and ease of computation: the larger the number of samples, the larger the memory requirement and the harder the computations. We ran BEL1D on the previous synthetic SNMR dataset with 10, 50, 100, 500, 1000, 5000, 10000,50000 and 100000 models in the prior samples. For each case, we ran BEL1D 10 times and aggregate the results. It is observed that the RMS error distribution tends to stabilize above 1000 models, suggesting that this value is optimal in our case (Figure 12), especially for the minimum and $5^{\text {th }}$ percentile values. This observation is confirmed by the evolution of the parameter distribution characteristics (Figure 12). In those graphs, the mean values of the parameters are normalized with the test values, and the standard deviations are normalized with the standard deviation in the prior model space. We see that the parameter distributions are stabilized above 1000 models, even if the values are not minimal. 

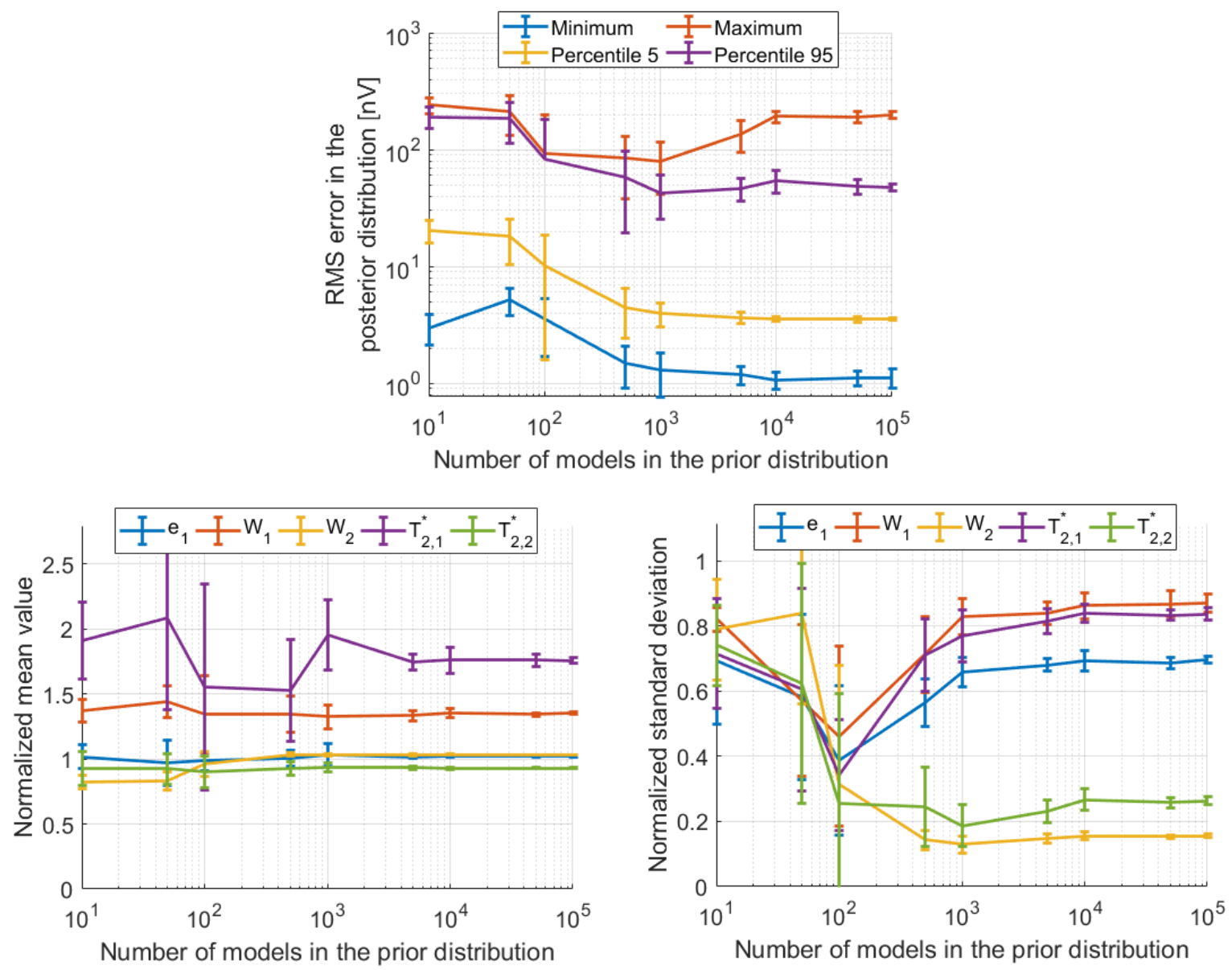

Figure 12 : Impact of the number of prior samples on the posterior distribution. The top graph presents RMS evolution, while the two bottom graphs show (from left to right) the normalized mean value and the normalized standard deviation.

To assess the impact of the kernel density bandwidth on the posterior distribution, we ran 10 times 10 different values ranging from $10^{-5}$ and 1 . In each case, 5000 models were sampled in the prior (Latin-hypercube sampler) and 5000 models were predicted, to ensure that no side effect is linked to other parameters. A similar analysis to the one performed in the previous subsection is then performed (Figure 13). low values result in erratic behaviors of the posterior distributions. On the other end, too large 482 bandwidths result in the smoothing of the posterior distributions, as is observed in the evolution 
483 of the standard deviation where this value increases sharply for the sensitive parameters.

484 Therefore, the optimal bandwidth is a value located in-between the two extremes, where the 485 reduction of uncertainty is significant for the sensitive parameters and the other parameters are 486 stable. This occurs around $10^{-2}$ in our case.

In our implementation of the kernel density estimator, the effect of a too small 488 bandwidth is avoided by verifying that enough points (at least $1 \%$ of the sampled prior) are 489 present in the direct surrounding of the reduced data $\left(\boldsymbol{d}_{\boldsymbol{o b s}}^{\boldsymbol{c}} \pm 3 \times\right.$ bandwidth). To perform the 490 test presented in this bandwidth analysis, this safeguarding measure was disabled. In the case 491 the default bandwidth is too small, then, the bandwidth is multiplied by 2 and the verification 492 is made again until the criterion is satisfied and only then, the kernel density estimation is 493 performed. We ran BEL1D with a bandwidth set to $10^{-5}$ and the safeguard algorithm enabled. 494 The obtained result is here similar to the one obtained with a suitable bandwidth as is shown by 495 the diamonds in the graph presenting the evolution of the normalized standard deviation in 496 Figure 13. 

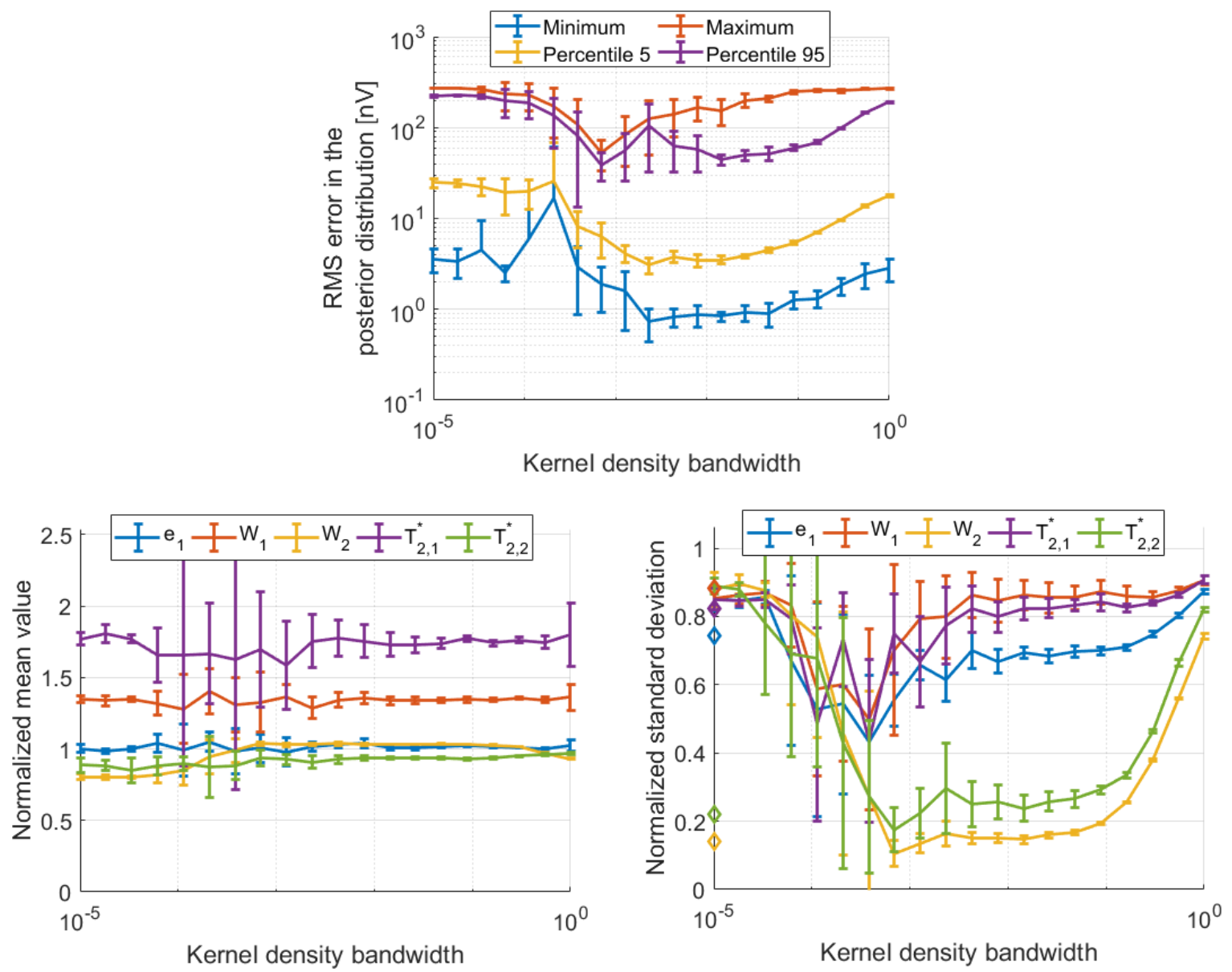

Figure 13 : Impact of the bandwidth in the kernel density estimation on the posterior distribution. The top graph presents RMS evolution, while the two bottom graphs show (from left to right) the normalized mean value and the normalized standard deviation. This latter also presents results obtained with the safeguarding measure enabled (diamonds) for the most extreme case: a bandwidth of $1 E-5$.

\section{Conclusion}

We propose an adaptation of the Bayesian Evidential Learning framework as a new tool

504 for the unidimensional interpretation of geophysical data called BEL1D. This approach offers 505 an alternative to stochastic inversions, as it requires fewer and simpler computations, thus 506 lowering the CPU cost. This approach is also presented as an alternative to deterministic 507 inversions as its behavior is superior both in terms of ease of implementation and completeness 508 of the results (handling properly non-uniqueness, quantifying the uncertainty, etc.). We therefore developed this method theoretically, as a new general algorithm. 
The framework does not require the use of regularization parameters or any other form

511 of bias, but rather requires the proper definition of the prior model space. Contrary to other

512 Bayesian methods, the use of a large prior model space (hence, introducing less bias in the

513 posterior information) does not involve heavier computations to converge as the algorithm is

514 always building in one step the posterior model space that corresponds to the observed data

515 (see, 2.4 Step 4: generation of posterior distributions for the model parameters in CCA space

516 using kernel density estimators). This method can therefore be considered unbiased if the prior

517 model space is sufficiently large. BEL1D is thus a computationally efficient alternative to

518 stochastic inversions as it only requires $n$ forward models corresponding to the prior samples.

519 Moreover, in BEL1D noise is considered via an imbedded theoretically based method, contrary

520 to classically used high regularization parameters that lack of theoretical basis to be properly

521 assessed and are therefore difficult to estimate.

When compared to McMC methods (such as DREAM), one of the key advantages of

BEL1D is the tractability of all operations. When McMC classically applies random changes to

524 the models and select them randomly, BEL1D constitutes a correlation between models

525 parameters and the data. This means that one could seek for the origin of all the posterior models

526 in the prior model space. This latter enables, for example, to efficiently sample multi-modal

527 distribution or to understand the origin of odd models in the posterior distribution (see the

528 numerical example), impossible to track back in McMC algorithms. The other key advantage

529 is the simpler tuning to convergence of BEL1D compared to DREAM (McMC). In DREAM,

530 it was required to change the dataspace to enable convergence towards reasonable (or even 531 correct) posterior distributions. In BEL1D, as long as the prior is correctly assumed, the only 532 parameter that the user needs to take care of is the number of models to sample in this space.

533 As shown in the discussion, this parameter can be selected by observing the stabilization of the 534 posterior distributions. In DREAM, the application without changes in the dataset 
representation lead to either nearly no reduction of uncertainty (using the RMS as a proxy of

536 the probability) or to a reduction towards level of uncertainty close to deterministic values but

537 without the true model inside the posterior (using all data points as proxy of the probability).

538 Therefore, applying this method to an unknown dataset might lead to erroneous interpretations,

539 whereas BEL1D, even if producing slightly larger uncertainties should always predict

540 consistent posterior spaces.

We demonstrated that BEL1D is efficient for the interpretation of SNMR data. We

542 presented results for noisy datasets originating from a numerical model and field. In both cases,

543 we validate the results from BEL1D, either against the test values from the model and McMC

544 methods - DREAM (Vrugt, 2016) - for the numerical example or other geophysical

545 experiments and classical state-of-the art inversion technics for the field case. On the other end,

546 the careful analysis of the RMS error of the proposed models indicates that the range of

547 uncertainty delivered by BEL1D is intrinsic to the non-unicity of the solution for geophysical

548 inverse problems: many models explain the data to the same level.

In term of performance, the computation cost of BEL1D is directly proportional to the efficiency of the forward model and the number of samples in the prior model space. It must be noted that the prior samples being independent, the simulation of synthetic data can be fully

552 parallelized. The performance of BEL1D can thus be easily estimated from the available 553 computing facilities. The cost is therefore much smaller than other stochastic methods requiring 554 ten to hundreds thousands of runs to converge towards a solution. Moreover, computation 555 timing could be dramatically reduced by pre-field forward modelling operations for large 556 geophysical campaigns where the prior would be similar. Prior realizations and the associated 557 datasets would then be reused (PCA and CCA operations also), letting only the kernel density 558 estimation, sampling and back-transformation operations to be applied after field acquisition, 
leading to extremely rapid imaging. This is an extreme advantage compared to other stochastic methods that rely on the data misfit (such as McMC) to sample the posterior.

This application, along with the pendulum case presented in supplementary material, demonstrate the versatility of BEL1D and its applicability to multiple cases with very few adaptations. Provided that a forward model already exists, the adaptation of the codes to any other 1D geophysical method would be straightforward. For example, the forward operator from HV-Inv (García-Jerez et al., 2016) could be used for the interpretation of H/V data. System calls may also help further extend the applicability of BEL1D. This may be the case for the use of all the 1D forward operators implemented in pyGIMLi (Rücker et al., 2017) - vertical electrical sounding, frequency- and time-domain electromagnetic and magnetotelluric.

In its present version, BEL1D is dedicated to the $1 \mathrm{D}$ inversion of geophysical data in layered Earth model where the contrast between layers is supposed to be sharp. In the future, we plan to extend the method to smoothly varying systems accounting for a large number of thin, correlated layers.

\section{Acknowledgment}

The authors would like to thank the contributors to the development of MRSmatlab, the cornerstone of SNMR data interpretation in BEL1D. Hadrien Michel is a fellow funded by the F.R.S.-FNRS.

\section{Computer codes availability}

The different MATLAB codes that have been used to produce the results presented in this paper are available at github.com/hadrienmichel/BEL1D. They are released with this paper under the BSD-2-Clause license. The codes consist in a series of graphical user interfaces for the interpretation of SNMR data (BEL1DSNMR), among others. All the functions are 
583 Computing toolbox is available.

Hadrien MICHEL

Urban and Environmental Engineering Department

$$
\text { B-4000 Liège }
$$

Belgium

(MRSMatlab: Müller-Petke et al., 2016).

\section{References}

Aster, R., Borchers, B., Thurber, C., 2013. Parameters estimation and inverse problems, 2nd ed. Academic Press.

Behroozmand, A.A., Auken, E., Fiandaca, G., Rejkjaer, S., 2016. Increasing the resolution and the signal-to-noise ratio of magnetic resonance sounding data using a central loop configuration. Geophysical Journal International 205, 243-256. https://doi.org/10.1093/gji/ggw004

Behroozmand, A.A., Keating, K., Auken, E., 2015. A Review of the Principles and Applications of the NMR Technique for Near-Surface Characterization. Surveys in Geophysics 36, 27-85. https://doi.org/10.1007/s10712-014-9304-0

Bobe, C., Van De Vijver, E., Keller, J., Hanssens, D., Van Meirvenne, M., De Smedt, P., 2019. Probabilistic One-Dimensional Inversion of Frequency-Domain Electromagnetic Data Using a Kalman Ensemble Generator. arXiv:1911.03275 [physics]. 
Bowman, A.W., Azzalini, A., 1997. Applied Smoothing Techniques for Data Analysis: The

Kernel Approach with S-Plus Illustrations, Oxford Statistical Science Series. Oxford University Press, Oxford, New York.

Devroye, L., 1986. Non-uniform random variate generation. Springer, New York.

García-Jerez, A., Piña-Flores, J., Sánchez-Sesma, F.J., Luzón, F., Perton, M., 2016. A computer code for forward calculation and inversion of the H/V spectral ratio under the diffuse field assumption. Computers \& Geosciences 97, 67-78. https://doi.org/10.1016/j.cageo.2016.06.016

Gilson, M., Briers, P., Ruthy, I., Dassargues, A., 2017. Elsenborn - Langert - Dreiherrenwald. Gorban, A.N., Kégl, B., Wunsch, D.C., Zinovyev, A.Y. (Eds.), 2008. Principal Manifolds for Data Visualization and Dimension Reduction, Lecture Notes in Computational Science and Enginee. Springer Berlin Heidelberg, Berlin, Heidelberg. https://doi.org/10.1007/978-3-540-73750-6

Hanssens, D., Delefortrie, S., Bobe, C., Hermans, T., De Smedt, P., 2019. Improving the reliability of soil EC-mapping: Robust apparent electrical conductivity (rECa) estimation in ground-based frequency domain electromagnetics. Geoderma 337, 11551163. https://doi.org/10.1016/j.geoderma.2018.11.030

Hermans, T., Lesparre, N., De Schepper, G., Robert, T., 2019. Bayesian evidential learning : a field validation using push-pull tests. Hydrogeology journal in press.

Hermans, T., Nguyen, F., Klepikova, M., Dassargues, A., Caers, J., 2018. Uncertainty Quantification of Medium-Term Heat Storage From Short-Term Geophysical Experiments Using Bayesian Evidential Learning. Water Resources Research 54, 29312948. https://doi.org/10.1002/2017WR022135

Hermans, T., Oware, E., Caers, J., 2016. Direct prediction of spatially and temporally varying physical properties from time-lapse electrical resistance data. Water Resources Research 52, 7262-7283. https://doi.org/10.1002/2016WR019126

Hertrich, M., Braun, M., Gunther, T., Green, A.G., Yaramanci, U., 2007. Surface Nuclear Magnetic Resonance Tomography. IEEE Transactions on Geoscience and Remote Sensing 45, 3752-3759. https://doi.org/10.1109/TGRS.2007.903829

Irving, J., Singha, K., 2010. Stochastic inversion of tracer test and electrical geophysical data to estimate hydraulic conductivities. Water Resources Research 46. https://doi.org/10.1029/2009WR008340

Jha, M.K., Kumar, S., Chowdhury, A., 2008. Vertical electrical sounding survey and resistivity inversion using genetic algorithm optimization technique. Journal of Hydrology 359, 71-87. https://doi.org/10.1016/j.jhydrol.2008.06.018

Kemna, A., Kulessa, B., Vereecken, H., 2002. Imaging and characterisation of subsurface solute transport using electrical resistivity tomography (ERT) and equivalent transport models. Journal of Hydrology 267, 125-146.

Kemna, A., Nguyen, F., Gossen, S., 2007. On linear model uncertainty computation in electrical imaging. Presented at the SIAM Conferance on Mathematical and Computational Issues in Geosciences, Santa Fe.

Krzanowski, W.J., 2000. Principles of multivariate analysis: A user's perspective, Revised Edition. ed, Oxford Statistical Series. Oxford University Press, New York.

LaBrecque, D.J., Miletto, M., Daily, W., Ramirez, A., Owen, E., 1996. The effects of noise on Occam's inversion of resistivity tomography data. Geophysics $61,538-548$.

Laloy, E., Hérault, R., Lee, J., Jacques, D., Linde, N., 2017. Inversion using a new lowdimensional representation of complex binary geological media based on a deep neural network. Advances in Water Resources 110, 387-405. https://doi.org/10.1016/j.advwatres.2017.09.029 
Laloy, E., Linde, N., Ruffino, C., Hérault, R., Gasso, G., Jacques, D., 2019. Gradient-based deterministic inversion of geophysical data with generative adversarial networks: Is it feasible? Computers \& Geosciences 104333. https://doi.org/10.1016/j.cageo.2019.104333

Lawrence, N.D., 2012. A Unifying Probabilistic Perspective for Spectral Dimensionality Reduction: Insights and New Models 30.

Lawyer, L.C., Bates, C., Rice, R., 2001. Geophysics in the affairs of mankind: a personalized history of exploration geophysics - 2nd edition, Society of Exploration Geophysics. ed. Tulsa, Oklahoma.

Lesparre, N., Nguyen, F., Kemna, A., Robert, T., Hermans, T., Daoudi, M., Flores-Orozco, A., 2017. A new approach for time-lapse data weighting in electrical resistivity tomography. Geophysics 82, E325-E333. https://doi.org/10.1190/geo2017-0024.1

Li, J., Lu, X., Farquharson, C.G., Hu, X., 2018. A finite-element time-domain forward solver for electromagnetic methods with complex-shaped loop sources. GEOPHYSICS 83, E117-E132. https://doi.org/10.1190/geo2017-0216.1

McKay, M.D., Beckman, R.J., Conover, W.J., 1979. A Comparison of Three Methods for Selecting Values of Input Variables in the Analysis of Output from a Computer Code. Technometrics 21, 239. https://doi.org/10.2307/1268522

Müller-Petke, M., Braun, M., Hertrich, M., Costabel, S., Walbrecker, J., 2016. MRSmatlab A software tool for processing, modeling, and inversion of magnetic resonance sounding data. GEOPHYSICS 81, WB9-WB21. https://doi.org/10.1190/geo20150461.1

Müller-Petke, M., Yaramanci, U., 2010. QT inversion - Comprehensive use of the complete surface NMR data set. GEOPHYSICS 75, WA199-WA209. https://doi.org/10.1190/1.3471523

Nguyen, F., Kemna, A., Robert, T., Hermans, T., 2016. Data-driven selection of the minimumgradient support parameter in time-lapse focused electric imaging. GEOPHYSICS 81, A1-A5. https://doi.org/10.1190/geo2015-0226.1

Park, J., Yang, G., Satija, A., Scheidt, C., Caers, J., 2016. DGSA: A Matlab toolbox for distance-based generalized sensitivity analysis of geoscientific computer experiments. Computers \& Geosciences 97, 15-29. https://doi.org/10.1016/j.cageo.2016.08.021

Phelps, G., Scheidt, C., Caers, J., 2018. Exploring viable geologic interpretations of gravity models using distance-based global sensitivity analysis and kernel methods. GEOPHYSICS 83, G79-G92. https://doi.org/10.1190/geo2017-0742.1

Rücker, C., Günther, T., Wagner, F.M., 2017. pyGIMLi: An open-source library for modelling and inversion in geophysics. Computers \& Geosciences 109, 106-123. https://doi.org/10.1016/j.cageo.2017.07.011

Sambridge, M., 2002. Monte Carlo methods in geophysical inverse problems. Reviews of Geophysics 40. https://doi.org/10.1029/2000RG000089

Satija, A., Caers, J., 2015. Direct forecasting of subsurface flow response from non-linear dynamic data by linear least-squares in canonical functional principal component space. Advances in Water Resources 77, 69-81. https://doi.org/10.1016/j.advwatres.2015.01.002

Scheidt, C., Li, L., Caers, J., 2018. Quantifying Uncertainty in Subsurface Systems, WileyBlackwell. ed.

Scheidt, C., Renard, P., Caers, J., 2015. Prediction-focused subsurface modeling: investigating the need for accuracy in flow-based inverse modeling. Mathematical Geosciences 47, 173-191. 
Socco, L.V., Foti, S., Boiero, D., 2010. Surface-wave analysis for building near-surface velocity models - Established approaches and new perspectives. GEOPHYSICS 75, 75A8375A102. https://doi.org/10.1190/1.3479491

Trainor-Guitton, W., Hoversten, G.M., 2011. Stochastic inversion for electromagnetic geophysics: Practical challenges and improving convergence efficiency. GEOPHYSICS 76, F373-F386. https://doi.org/10.1190/geo2010-0223.1

Vrugt, J.A., 2016. Markov chain Monte Carlo simulation using the DREAM software package: Theory, concepts, and MATLAB implementation. Environmental Modelling \& Software 75, 273-316. https://doi.org/10.1016/j.envsoft.2015.08.013

Wand, M.P., Jones, M.C., 1993. Comparison of Smoothing Parameterizations in Bivariate Kernel Density Estimation. Journal of the American Statistical Association 88, 520. https://doi.org/10.2307/2290332

Wastiaux, C., 2008. Les tourbières sont-elles des éponges régularisant l'écoulement? Bulletin de la Société géographique de Liège 50.

Wastiaux, C., Schumacker, R., 2003. Topographie de surface et de subsurface des zones tourbeuses des réserves naturelles domaniales des Hautes-Fagnes (No. C60/1-2-3). Université de Liège, Service de phytosociologie, flore et végétation des Hautes-Fagnes.

Yang, F., Ma, J., 2019. Deep-learning inversion: A next-generation seismic velocity model building method. GEOPHYSICS 84, R583-R599. https://doi.org/10.1190/geo20180249.1 